\section{ORIGINAL RESEARCH}

\author{
H. Matsuda \\ S. Mizumura \\ K. Nemoto \\ F. Yamashita \\ E. Imabayashi \\ N. Sato \\ T. Asada
}

\title{
Automatic Voxel-Based Morphometry of Structural MRI by SPM8 plus Diffeomorphic Anatomic Registration Through Exponentiated Lie Algebra Improves the Diagnosis of Probable Alzheimer Disease
}

\begin{abstract}
BACKGROUND AND PURPOSE: The necessity for structural MRI is greater than ever to both diagnose $A D$ in its early stage and objectively evaluate its progression. We propose a new VBM-based software program for automatic detection of early specific atrophy in AD.
\end{abstract}

\begin{abstract}
MATERIALS AND METHODS: A target VOI was determined by group comparison of 30 patients with very mild $A D$ and 40 age-matched healthy controls by using SPM. Then this target VOI was incorporated into a newly developed automated software program independently running on a Windows PC for VBM by using SPM8 plus DARTEL. ROC analysis was performed for discrimination of 116 other patients with $\mathrm{AD}$ with very mild stage $(n=45)$, mild stage $(n=30)$ and moderate-to-advanced stages ( $n=41)$ from 40 other age-matched healthy controls by using a $z$ score map in the target VOI.
\end{abstract}

\begin{abstract}
RESULTS: Medial temporal structures involving the entire region of the entorhinal cortex, hippocampus, and amygdala showed significant atrophy in the patients with very mild AD and were determined as a target VOI. When we used the severity score of atrophy in this target VOI, $91.6 \%, 95.8 \%$, and $98.2 \%$ accuracies were obtained in the very mild $A D$, mild $A D$, and moderate-to-severe $A D$ groups, respectively. In the very mild $A D$ group, a high specificity of $97.5 \%$ with a sensitivity of $86.4 \%$ was obtained, and age at onset of AD did not influence this accuracy.
\end{abstract}

CONCLUSIONS: This software program with application of SPM8 plus DARTEL to VBM provides a high performance for AD diagnosis by using MRI.

ABBREVIATIONS: $A D=$ Alzheimer disease; $D A R T E L=$ diffeomorphic anatomical registration through exponentiated lie algebra; FWHM = full width at half maximum; $\mathrm{MCl}=$ mild cognitive impairment; MMSE = Mini-Mental State Examination; $R O C=$ receiver operating characteristic analysis; SPM = statistical parametric mapping; VBM = voxel-based morphometry

ncreases in the number of individuals with dementia, the highest proportion of whom are affected by $\mathrm{AD}$, have made early diagnosis of $\mathrm{AD}$ a major research and clinical priority. Of several neuroimaging techniques that provide surrogate markers for the diagnosis of $\mathrm{AD}$, structural MRI is the most commonly used because of its noninvasiveness and excellent spatial resolution with good tissue contrast. ${ }^{1}$ In $\mathrm{AD}$, the earliest tissue loss occurs in the medial temporal structures, particularly in the entorhinal cortex. ${ }^{2}$ However, visual inspection is

\section{Received August 26, 2011; accepted after revision September 23.}

From the Department of Nuclear Medicine (H.M., E.I.), Saitama Medical University International Medical Center, Hidaka, Saitama, Japan; Department of Radiology (S.M.), Toho University Omori Medical Center, Ota-ku, Tokyo, Japan; Department of Psychiatry (K.N., T.A.), Graduate School of Comprehensive Human Sciences, University of Tsukuba, Tsukuba, Ibaraki, Japan; Research Association for Biotechnology (F.Y.), Minato-ku, Tokyo, Japan; and Department of Radiology (N.S.), National Center Hospital, National Center of Neurology and Psychiatry, Kodaira, Tokyo, Japan.

This work was supported by a Grant-in-Aid for Scientific Research, Ministry of Education, Culture, Sports, Science and Technology, Japan (21591578).

Please address correspondence to Hiroshi Matsuda, MD, Department of Nuclear Medicine, Saitama Medical University International Medical Center, 1397-1, Yamane Hidaka, Saitama, Japan; e-mail: matsudah@saitama-med.ac.jp

Indicates open access to non-subscribers at www.ajnr.org

Indicates article with supplemental on-line tables.

http://dx.doi.org/10.3174/ajnr.A2935 insufficient for objective evaluation of mild atrophy. Although manual tracing of these structures can quantify the absolute volume, it is time-consuming and requires special expertise in anatomic knowledge for tracers. Recently, computer-aided $\mathrm{VBM}^{3}$ has been applied to detect early atrophic changes in $\mathrm{AD}$. Although this technique cannot provide the absolute volume, it can provide statistical results in comparisons of patients with $\mathrm{AD}$ with healthy controls. ${ }^{4}$ Moreover VBM has been reported to be a surrogate indicator of the full brain topographic representation of the neurodegenerative aspect of $\mathrm{AD}$ pathology. ${ }^{5}$ Hirata et $\mathrm{al}^{6}$ proposed an automated software program, a voxel-based specific regional analysis system for $\mathrm{AD}$, for the diagnosis of $\mathrm{AD}$ by using this VBM technique. In the present study, we revised this software by introducing new techniques and validated its utility.

\section{Materials and Methods}

A total of 251 subjects were studied in 1 center. We retrospectively chose 146 patients ( 65 men and 81 women) with a clinical diagnosis of probable AD according to the National Institute of Neurologic and Communicative Disorders and Stroke and the Alzheimer Disease and Related Disorders Association criteria. ${ }^{7}$ These patients were classified into 3 groups of very mild, mild, and moderate-to-advanced $\mathrm{AD}$. The very mild $\mathrm{AD}$ group comprised 75 patients ( 37 men and 38 women) who ranged in age from 51 to 86 years with a mean of $71.2 \pm 7.4$ years. 
At the initial visit, they had no apparent loss in general cognitive, behavioral, or functional status and corresponded to the criteria of the amnestic type of $\mathrm{MCI}^{8}$ or 0.5 in the Clinical Dementia Rating. ${ }^{9}$ The MMSE score ranged from 24 to 29 (mean, $25.7 \pm 1.5$ ). During the subsequent follow-up period of 2-6 years, the subjects showed progressive cognitive decline and eventually fulfilled the diagnosis of probable $\mathrm{AD}$. The mild and moderate-to-advanced $\mathrm{AD}$ groups comprised 30 patients ( 8 men and 22 women, $71.4 \pm 6.8$ years of age) and 41 patients ( 20 men and 21 women, $71.3 \pm 7.7$ years of age), respectively. The MMSE score ranged from 20 to 25 (mean, $21.4 \pm 1.3$ ) and from 6 to 19; (mean, $15.0 \pm 3.5$ ) for the mild and moderate-toadvanced $\mathrm{AD}$ groups, respectively. Eighty-one of these patients with $\mathrm{AD}$ (48 very mild, 11 mild, and 22 moderate-to-advanced) underwent follow-up MRI studies at an interval of 1-4 years for, at most, 6 years (mean, $3.3 \pm 1.2$ years), and most patients in the very mild and mild $\mathrm{AD}$ groups moved to a more advanced group during the follow-up period. Consequently, the total of MRI studies was 89, 57, and 123 for the very mild, mild, and moderate-to-advanced $\mathrm{AD}$ groups.

Eighty age-matched control subjects (37 men and 43 women) were healthy volunteers with no memory impairment or cognitive disorders. They ranged in age from 54 to 86 years with a mean of $70.4 \pm 7.8$ years. Their performance was within normal limits both on the Wechsler Memory Scale-Revised and the Wechsler Adult Intelligence Scale-Revised. Their MMSE scores ranged from 26 to 30 (mean, $29.1 \pm 1.2$ ). They did not differ in age or education from the patients with $\mathrm{AD}$. Additionally, 25 healthy volunteers ( 15 men and 10 women; mean, $31.1 \pm 7.8$ years of age) participated in this study for creation of a customized template for spatial normalization in the statistical image analysis. The ethics committee approved this study, and all subjects provided informed consent to participate. None of them had asymptomatic cerebral infarction detected by T2-weighted MRI.

All subjects underwent an MRI study on a 1.5T Vision Plus imager (Siemens, Erlangen, Germany). One hundred forty 3D sections of a T1-weighted magnetization-prepared rapid acquisition of gradient echo sequence were obtained in a sagittal orientation as $1.2-\mathrm{mm}$ thick sections $\left(\mathrm{FOV}=23, \mathrm{TR}=9.7 \mathrm{~ms}, \mathrm{TE}=4 \mathrm{~ms}\right.$, flip angle $=12^{\circ}$, and $\mathrm{TI}=300 \mathrm{~ms}$, with no intersection gaps).

First, to define a target VOI for early diagnosis of $\mathrm{AD}$, we performed a group comparison between 30 patients ( 14 men and 16 women; mean age, $73.8 \pm 4.8$ years) randomly chosen in the present very mild $\mathrm{AD}$ group and the present 40 healthy controls group (19 men and 21 women; mean age, $70.8 \pm 8.5$ years). Using the latest version of SPM8 (Wellcome Department of Imaging Neuroscience, London, United Kingdom), we segmented MRIs into gray matter, white matter, and CSF images by a unified tissue-segmentation procedure after image-intensity nonuniformity correction. These segmented gray matter images were then spatially normalized to the customized template in the standardized anatomic space by using DARTEL (Wellcome Department of Imaging Neuroscience). ${ }^{10}$ The customized template for DARTEL was created from the aforementioned 25 healthy young subjects. To preserve gray matter volume within each voxel, we modulated the images by the Jacobean determinants derived from the spatial normalization by DARTEL and then smoothed them by using an 8-mm FWHM Gaussian kernel. To compare the present analysis by using SPM8 plus DARTEL with the previously reported analysis, ${ }^{6}$ we also defined a target VOI by using an old SPM version, SPM2 (Wellcome Department of Imaging Neuroscience) between the same 2 groups. Group comparisons by SPM were assessed by using the family-wise error at a threshold of $P<.05$, corrected for multiple comparisons.

A stand-alone software program running on Windows for VBM analysis by SPM8 plus DARTEL was developed to discriminate patients with AD from healthy controls. First, MRIs were spatially normalized with only a 12-parameter affine transformation to the SPM template so as to correct for differences in brain size. These linearly transformed images were nonlinearly transformed and then modulated to the customized template for DARTEL, followed by smoothing by using an 8-mm FWHM kernel. Each processed gray matter image of the remaining 116 patients with $\mathrm{AD}$ and 40 healthy controls was compared with the mean and SD of gray matter images of the 40 healthy volunteers chosen in the group comparison by using voxelby-voxel $z$ score analysis with and without voxel normalization to global mean intensities (global normalization): $z$ score $=([$ control mean] - [individual value]) / (control SD). These $z$ score maps were displayed by overlay on tomographic sections and surface rendering of the standardized brain. This program registered the target VOI defined by the aforementioned group comparison. This software program takes 8 minutes 40 seconds for all procedures by using a 64-bit $\mathrm{PC}$ with a Core i7 central processing unit and 6-gigabytes memory (Intel, Santa Clara, California).

We determined 4 indicators for characterizing atrophy in a target VOI and in the whole brain: first, the severity of atrophy obtained from the averaged positive $z$ score in the target VOI; second, the extent of a region showing significant atrophy in the target VOI- that is, the percentage rate of the coordinates with a $z$ value exceeding the threshold value of 2 in the target VOI; third, the extent of a region showing significant atrophy in the whole brain - that is, the percentage rate of the coordinates with a $z$ value exceeding the threshold value of 2 in the whole brain; and fourth, the ratio of the extent of a region showing significant atrophy in the target VOI to the extent of a region showing significant atrophy in the whole brain. The utility of these indicators for this discrimination of $\mathrm{AD}$ from healthy controls has been reported in previous MRI ${ }^{6}$ and SPECT studies. ${ }^{11}$

These 4 indicators were obtained under 2 conditions, with or without global normalization. Using the values of the 4 indicators as the threshold, we determined ROC curves for discrimination of patients with $\mathrm{AD}$ from healthy volunteers by using JMP 7.0 (SAS Institute, Cary, North Carolina). The program calculates the area under the ROC curves, sensitivity, specificity, and accuracy. Moreover, the age effects of $\mathrm{AD}$ onset on these 4 indicators and the results of the ROC were investigated in the very mild $\mathrm{AD}$ group classified into 2 subgroups with an age threshold of 65: the early-onset subgroup (16 patients, 9 men and 7 women; mean age, $58.0 \pm 4.6$ years) and the late-onset subgroup ( 29 patients, 14 men and 15 women; mean age, $73.8 \pm 4.4$ years $)$.

\section{Results}

The group comparison by SPM8 plus DARTEL demonstrated significant decline of gray matter volume in the left (Talairach coordinates $-24,-10,-14, \mathrm{x}, \mathrm{y}, \mathrm{z} ; z=7.37$ and 6.95 without and with global normalization, respectively) and the right $(24,10,14, x, y, z ; z=7.42$ and 7.05 without and with global normalization, respectively) parahippocampal gyri in patients with very mild $\mathrm{AD}$ (Fig 1). These bilateral regions involve the entorhinal cortex, head to tail of the hippocampus, and amygdala and are delineated as a target VOI for AD. Group comparison by SPM 2 showed significant decline of gray matter volume in the left $(-18,-7,-16, \mathrm{x}, \mathrm{y}, \mathrm{z} ; z=6.18)$ and right 


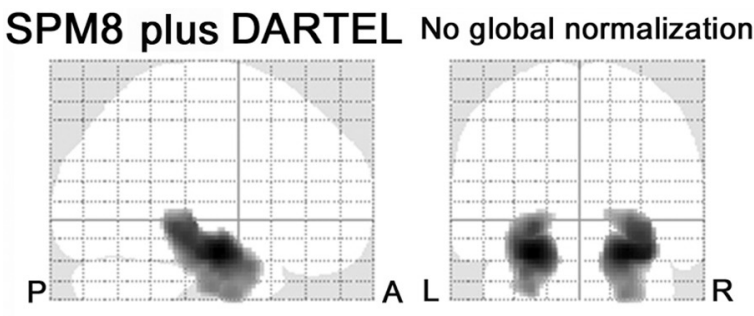

SPM8 plus DARTEL Global normalzation

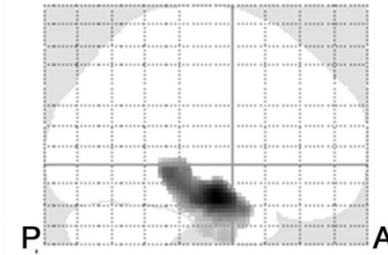

\section{SPM2}

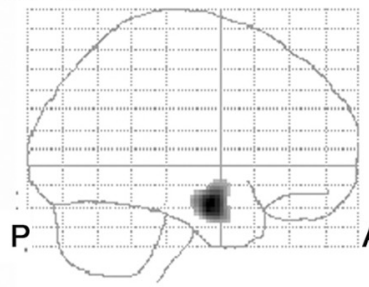

A L

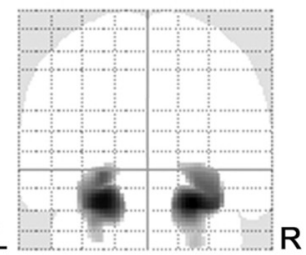

Global normalzation

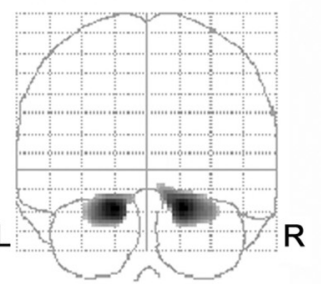

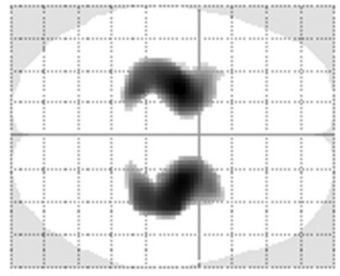

$\nabla$

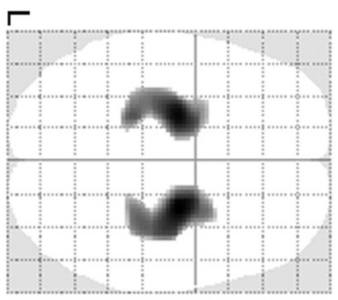

D

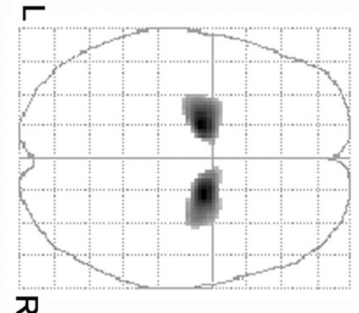

Fig 1. Group comparison of gray matter volume by SPM8 plus DARTEL and SPM2 between 30 patients with very mild AD and 40 healthy age-matched volunteers. The SPM8 plus DARTEL analysis demonstrates significant decline of gray matter volume in the bilateral medial temporal structures both with and without global normalization in patients with very mild AD. The cluster shape is very close to the anatomic configuration of the medial temporal structures involving the entorhinal cortex, amygdala, and hippocampal formation from head to tail. Although the SPM 2 analysis demonstrates a significant decline of gray matter volume in the bilateral medial temporal structures, the cluster is confined to the anterior parts of the medial temporal structures.

\begin{tabular}{|c|c|c|c|c|c|c|c|c|c|}
\hline \multirow[b]{3}{*}{ Group } & \multirow{3}{*}{$\begin{array}{c}\text { Global } \\
\text { Normalization }\end{array}$} & \multicolumn{4}{|c|}{ SPM8 plus DARTEL } & \multicolumn{4}{|c|}{ SPM2 } \\
\hline & & \multicolumn{3}{|c|}{ Target VOI } & \multirow{2}{*}{$\begin{array}{c}\text { Whole- } \\
\text { Brain } \\
\text { Extent }(\%)\end{array}$} & \multicolumn{3}{|c|}{ Target VOI } & \multirow{2}{*}{$\begin{array}{l}\text { Whole- } \\
\text { Brain } \\
\text { Extent (\%) }\end{array}$} \\
\hline & & Severity & Extent $(\%)$ & Ratio & & Severity & Extent $(\%)$ & Ratio & \\
\hline \multirow[t]{2}{*}{ Healthy controls } & - & $0.7 \pm 0.5$ & $4.4 \pm 9.8$ & $0.8 \pm 1.5$ & $2.5 \pm 4.7$ & NA & NA & NA & NA \\
\hline & + & $0.7 \pm 0.3$ & $2.0 \pm 4.9$ & $1.3 \pm 2.8$ & $1.4 \pm 0.9$ & $0.5 \pm 0.3$ & $1.8 \pm 7.3$ & $0.5 \pm 1.8$ & $2.6 \pm 3.1$ \\
\hline \multirow[t]{2}{*}{ Very mild $A D$} & - & $1.8 \pm 0.9^{b}$ & $39.0 \pm 35.5^{b}$ & $9.9 \pm 8.9^{b}$ & $5.4 \pm 7.6^{b}$ & NA & NA & NA & NA \\
\hline & + & $2.2 \pm 0.9^{b}$ & $49.2 \pm 30.2^{b}$ & $12.9 \pm 7.8^{b}$ & $4.1 \pm 2.5^{b}$ & $1.6 \pm 1.0^{b}$ & $30.8 \pm 32.1^{\mathrm{b}}$ & $6.7 \pm 7.8^{b}$ & $5.4 \pm 3.7^{b}$ \\
\hline \multirow[t]{2}{*}{ Mild AD } & - & $2.2 \pm 0.7^{b}$ & $53.7 \pm 29.8^{b}$ & $12.8 \pm 8.8^{b}$ & $5.5 \pm 5.1^{b}$ & NA & NA & NA & NA \\
\hline & + & $2.7 \pm 0.8^{\mathrm{b}}$ & $63.7 \pm 25.8^{b}$ & $15.4 \pm 7.8^{b}$ & $4.3 \pm 1.9^{b}$ & $2.1 \pm 1.1^{\mathrm{b}}$ & $42.0 \pm 32.3^{b}$ & $9.6 \pm 9.2^{b}$ & $5.4 \pm 3.0^{b}$ \\
\hline \multirow{2}{*}{$\begin{array}{l}\text { Moderate-to-advanced } \\
\text { AD }\end{array}$} & - & $2.8 \pm 1.0^{b, c, d}$ & $72.2 \pm 26.5^{b, c, d}$ & $8.4 \pm 7.2^{b}$ & $15.1 \pm 14.0^{b, c, d}$ & NA & NA & NA & NA \\
\hline & + & $3.0 \pm 1.0^{\mathrm{b}, \mathrm{c}}$ & $68.7 \pm 24.1^{\mathrm{b}, \mathrm{c}}$ & $11.7 \pm 6.7^{\mathrm{b}, \mathrm{d}}$ & $7.1 \pm 3.7^{\mathrm{b}, \mathrm{c}, \mathrm{d}}$ & $2.6 \pm 1.4^{b, c}$ & $56.3 \pm 33.2^{b . c}$ & $7.6 \pm 6.2^{b}$ & $9.0 \pm 5.0^{b, c, d}$ \\
\hline
\end{tabular}

Note:-NA indicates not applicable; +, presence; - , absence.

a Tukey honest significance test in each condition of global normalization.

b $P<.001$ versus healthy controls.

${ }^{\mathrm{c}} P<.001$ versus very mild $\mathrm{AD}$ group.

${ }^{d} P<.001$ versus mild $A D$ group.

$(18,-5,15, \mathrm{x}, \mathrm{y}, \mathrm{z} ; z=5.86)$ parahippocampal gyri (Fig 1). The cluster size was smaller in SPM2 than in SPM8 plus DARTEL.

The patients with AD showed significantly $(P<.001$, Tukey honest significance test) greater values than healthy controls in all 4 indicators in both SPM8 plus DARTEL and SPM2 analysis (Table 1). The mild AD group showed not significant but greater values of all 4 indicators than the very mild AD group. The moderate-to-advanced AD group showed significantly $(P<.001)$ greater values of severity and extent for the target VOI and extent for the whole brain than the very mild $\mathrm{AD}$ group. In contrast, the ratio for the target $\mathrm{VOI}$ in the moderate-to-advanced $\mathrm{AD}$ group was lower than that in the mild $\mathrm{AD}$ group and almost equal to that in the very mild $\mathrm{AD}$ group. Global normalization in SPM8 plus DARTEL analysis elevated the severity and ratio for the target VOI and diminished the extent for the whole brain in all $\mathrm{AD}$ groups. The SPM2 analysis showed lower values in severity, extent, and ratio for a target VOI and greater values in extent for the whole brain than the SPM8 plus DARTEL analysis.

In the SPM8 plus DARTEL analysis, better ROC results were obtained in the condition with than without global normalization, particularly in specificity (On-line Table 1). Of the 


\begin{tabular}{|c|c|c|c|c|c|c|c|c|c|}
\hline \multirow{3}{*}{$\begin{array}{l}\text { Global } \\
\text { Normalization }\end{array}$} & \multirow[b]{3}{*}{ Onset } & \multicolumn{4}{|c|}{ SPM8 plus DARTEL } & \multicolumn{4}{|c|}{ SPM2 } \\
\hline & & \multicolumn{3}{|c|}{ Target VOI } & \multirow{2}{*}{$\begin{array}{l}\text { Whole- } \\
\text { Brain } \\
\text { Extent }(\%)\end{array}$} & \multicolumn{3}{|c|}{ Target VOI } & \multirow{2}{*}{$\begin{array}{c}\text { Whole- } \\
\text { Brain } \\
\text { Extent } 1 \%\end{array}$} \\
\hline & & Severity & Extent $(\%)$ & Ratio & & Severity & Extent $(\%)$ & Ratio & \\
\hline- & Early & $1.5 \pm 0.7$ & $25.3 \pm 31.1$ & $3.8 \pm 3.8$ & $5.1 \pm 4.9$ & NA & NA & NA & NA \\
\hline- & Late & $1.9 \pm 0.9$ & $43.8 \pm 35.6$ & $5.9 \pm 8.4$ & $11.6 \pm 9.5$ & NA & NA & NA & NA \\
\hline+ & Early & $1.9 \pm 0.7$ & $37.4 \pm 26.2$ & $9.5 \pm 6.0$ & $4.1 \pm 1.7$ & $1.4 \pm 1.0$ & $24.4 \pm 34.6$ & $4.7 \pm 5.5$ & $3.8 \pm 2.4$ \\
\hline+ & Late & $2.3 \pm 0.9$ & $53.6 \pm 30.7$ & $14.1 \pm 8.1$ & $4.1 \pm 2.7$ & $1.7 \pm 1.0$ & $33.2 \pm 31.2$ & $7.5 \pm 8.5$ & $6.0 \pm 3.9$ \\
\hline
\end{tabular}

Note:-NA indicates not applicable; +, presence; -, absence.

4 indicators, the severity and extent for the target VOI with global normalization showed almost equal and high accuracy. Even in the very mild AD group, the severity showed a high accuracy of $91.6 \%$, increasing to $95.8 \%$ in the mild AD group and $98.2 \%$ in the moderate-to-advanced AD group. SPM8 plus DARTEL showed better ROC results for all 4 indicators than SPM2.

Although the early-onset subgroup showed lower values of indicators for the target VOI than the late-onset subgroup (Table 2), global normalization elevated these indicators evenly in the early- and late-onset subgroups. These indicators were largely stable before and after global normalization in healthy controls. Consequently, ROC results in SPM8 plus DARTEL revealed equal accuracy after global normalization between these 2 subgroups (Online Table 2). In contrast, SPM2 analysis showed approximately 10\% lower accuracy in the early-onset subgroup than that in the late-onset subgroup when using the severity score.

In each of 81 follow-up patients with $\mathrm{AD}$, the severity score in a target VOI gradually increased from the baseline to follow-up studies. The annual increase of the severity score after global normalization was $0.27 \pm 0.15$.

Representative cross-sectional and longitudinal studies for
SPM8 plus DARTEL analysis are demonstrated in Figs 2 and 3, respectively.

\section{Discussion}

Using severity as an indicator, we obtained a high sensitivity of $86.4 \%$ and extremely high specificity of $97.5 \%$, resulting in an accuracy of $91.6 \%$ for discrimination of patients with very mild AD from healthy controls in the SPM8 plus DARTEL analysis. Extremely high specificity mainly contributed to this high accuracy. In the SPM2 analysis, ROC analysis presented $5 \%$ lower sensitivity and approximately $13 \%$ lower specificity compared with those in the SPM8 plus DARTEL analysis. Kawachi et $\mathrm{al}^{12}$ reported $82.9 \%$ in both sensitivity and specificity in the patients with very mild AD in a similar VBM study by using an older SPM version, SPM99. This better specificity may result from application of the SPM8 plus DARTEL algorithm. This DARTEL algorithm can provide more precise spatial normalization to the template than the conventional algorithm. $^{10,13}$

This improvement in the preciseness of the spatial normalization was confirmed by group comparison for determining a target VOI. The SPM8 plus DARTEL results showed significantly decreased volume with anatomically precise configura-

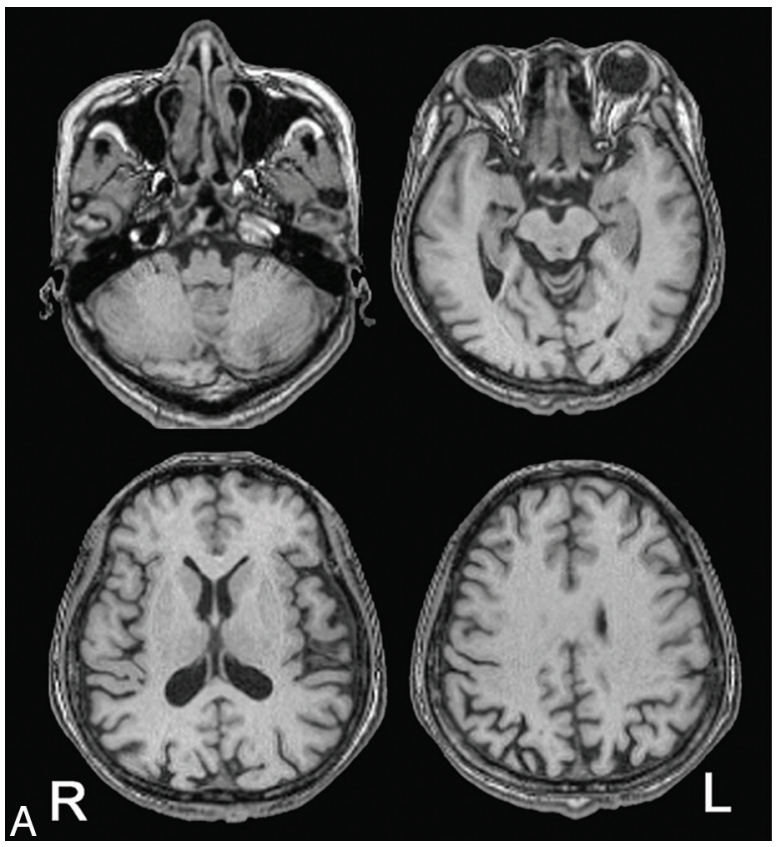

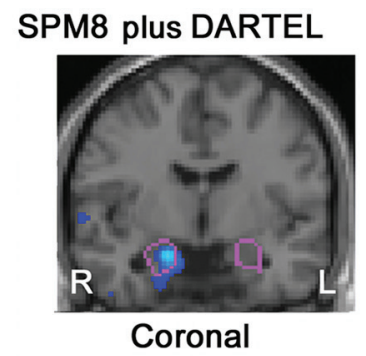
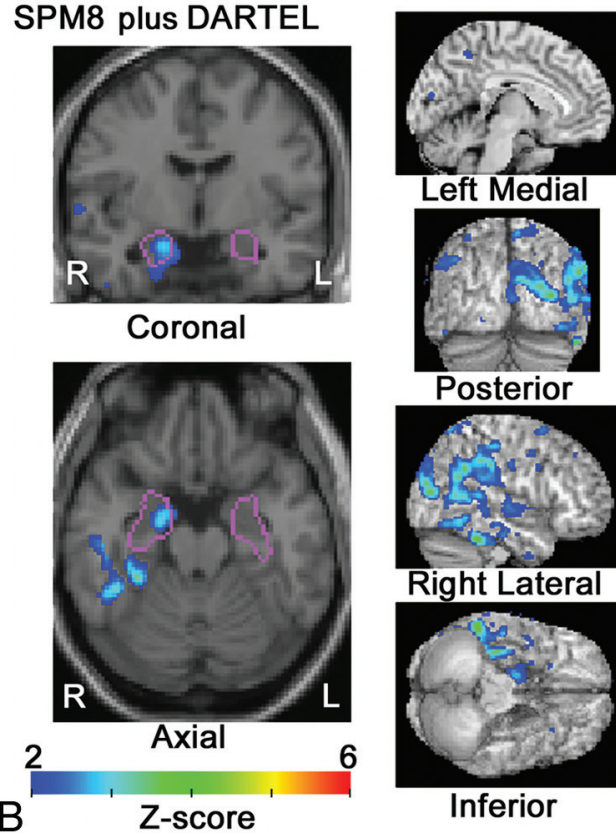

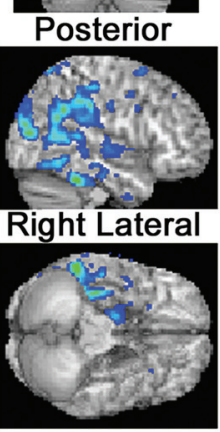

Inferior

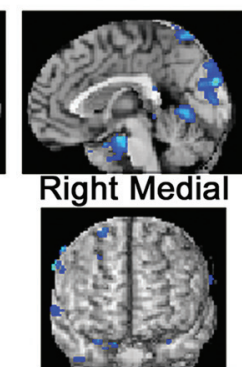

Anterior
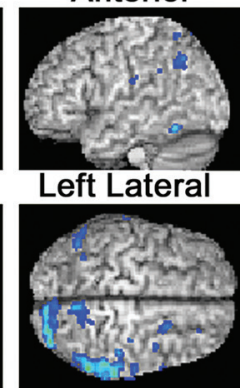

Superior

Fig 2. Cross-sectional VBM study by using SPM8 plus DARTEL. $A$, MR image of a 52-year-old woman with an MMSE score of 27. One year later the MMSE score declined to 19. $B$, SPM8 plus DARTEL analysis with global normalization reveals a significant decrease of gray matter volume in the right entorhinal area. Colored areas with $z$ scores of $>2$ are overlaid as significantly atrophied regions on tomographic sections and cortical surface of the standardized MRI template. A target VOI in the medial temporal structures is demarcated with purple lines. The right temporoparietal cortex also shows extensive significant atrophy. 


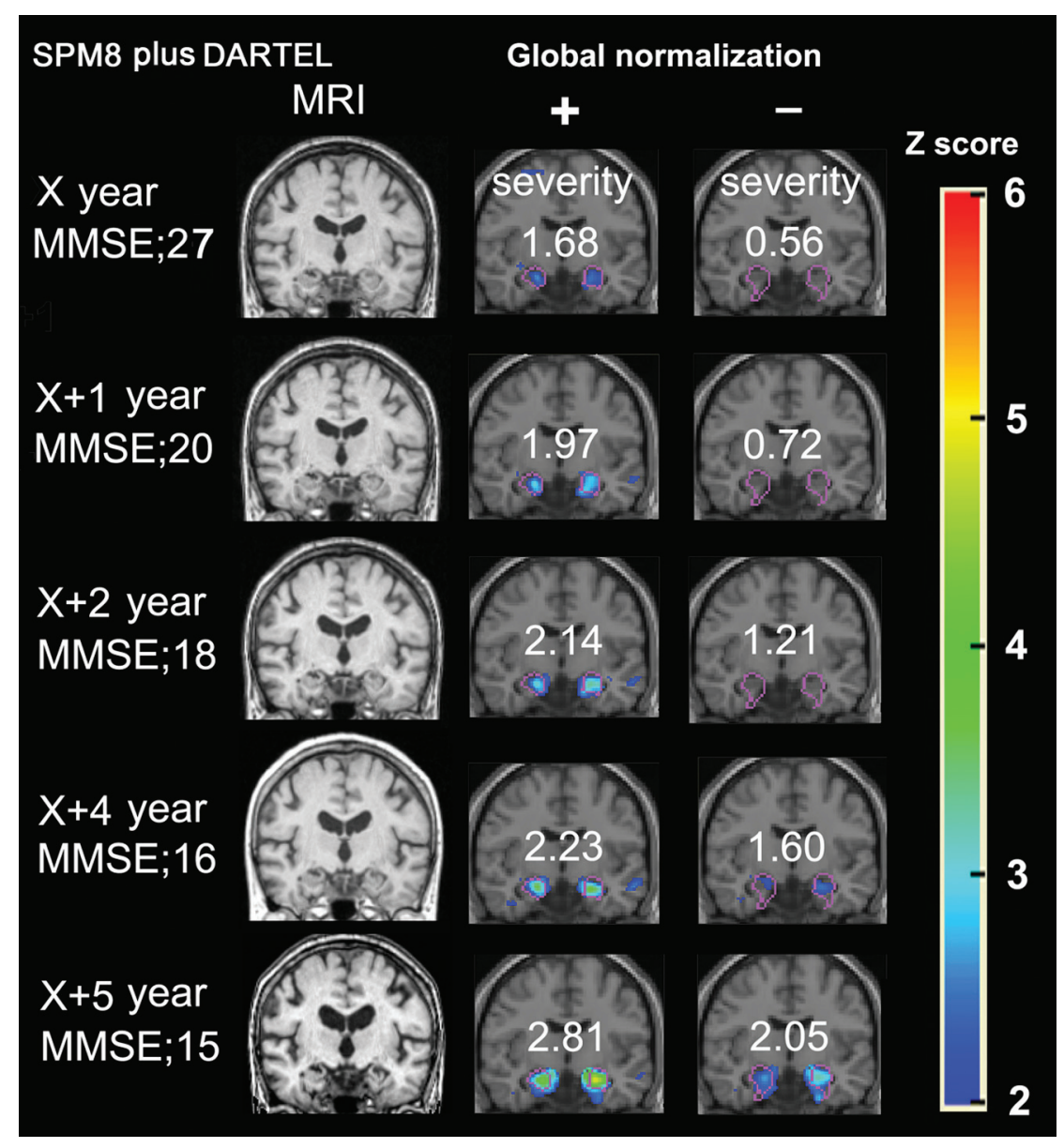

Fig 3. Longitudinal VBM studies by using SPM8 plus DARTEL. A 63-year-old woman with an MMSE score of 27 at the first visit was followed up for 6 years. One year later, the MMSE score decreased to 20 and gradually decreased thereafter. VBM analysis with global normalization reveals significant atrophy in the bilateral medial temporal areas even at the time of the initial study. Then the $z$ score in a target Vol increased step by step with time. In contrast, analysis without global normalization does not demonstrate significant atrophy in the medial temporal areas for the first 3 years. Severity scores as an indicator for characterizing atrophy in the medial temporal structures are shown.

tion of the medial temporal structures involving the entorhinal cortex, amygdala, and total hippocampal formation. Takahashi et $\mathrm{al}^{14}$ demonstrated almost identical results by using SPM8 plus DARTEL. The present SPM results by using conventional VBM by SPM2 showed decreased gray matter volume mainly in the anterior parts of the parahippocampal gyri with a less precise configuration. The severity score proved to be useful for longitudinal studies as well. The annual increase of this score may be indicative of disease progression.

The modulation in VBM allows comparison of the absolute amount of gray matter. ${ }^{15}$ The step of global normalization allows correction of the absolute amount of gray matter for individual total brain volume. Comparison of discrimination performance demonstrated better results in the condition with than without global normalization. This difference in discrimination performance may arise from the well-known fact of selective atrophy in the medial temporal structures in AD. ${ }^{1,4-6,16,17}$ Even if the absolute amount of gray matter of the medial temporal structures is decreased, a concomitant decrease in the total volume of gray matter would decrease specificity. The specificity in ROC analysis of the severity for the target VOI was 17\% lower in the condition without than with global normalization in very mild $\mathrm{AD}$. The degree of selective atrophy in the medial temporal areas can be assessed by the ratio as an indicator. In patients with $\mathrm{AD}$, more than 10 -fold selective atrophy was observed in the medial temporal areas compared with the whole brain in SPM8 plus DARTEL. Global normalization enhanced this ratio. Progression of neocortical atrophy would result in the decline of this ratio in advanced $\mathrm{AD}$. This indicator may be useful for differentiation of $\mathrm{AD}$ from other neuropsychiatric diseases manifesting dementia.

The early-onset subgroup showed milder atrophy in the medial temporal structures than the late-onset subgroup. This is in line with several previous reports in which lateonset subgroups showed greater atrophy in the medial temporal structures than the early-onset subgroups. ${ }^{16,17}$ However global normalization in SPM8 plus DARTEL extended the difference of indicators for a target VOI evenly in the early-onset and late-onset subgroups from indicators in healthy controls. This extension led to almost equal accuracy for discrimination of early-onset and late-onset very mild AD from healthy controls. This global normalization procedure may make it possible to use a common target VOI irrespective of age at onset of AD.

Thus the present study made it clear that the global normalization procedure in VBM by using SPM8 plus DARTEL has advantages in enhancing the discrimination power of diagnosing $\mathrm{AD}$. However lower values of the extent for the whole brain after global normalization would underestimate neocor- 
tical atrophy. The extent for the whole brain without global normalization may be useful for accurately evaluating the degree of neocortical atrophy.

This study is not without limitations. First, we should investigate whether this 1-site study is applicable to multicenter studies. Second, evaluation of the reproducibility of the present VBM technique may be necessary for longitudinal studies. Third, we investigated patients with amnestic MCI who all converted to AD. The outcome for any patient with MCI is uncertain because many subjects remain stable or even revert to a normal state, while others progress to dementia. Accordingly, the predictive study by using this VBM approach is much more important for MCI conversion to AD. Fourth, the single target VOI was used irrespective of age at onset of AD. A similar VBM study by Ishii et $\mathrm{al}^{18}$ recommended the use of a target VOI involving not only medial temporal structures but also parietal and posterior cingulate cortices and precunei in early-onset AD. Although this software program presented the same accuracy between early- and late-onset very mild $\mathrm{AD}$ subgroups, we may have to investigate a more appropriate target VOI from a larger number of patients with early-onset AD. However, incorporation of 2 types of target VOIs for early- and late-onset AD into a software program may confound the program user in the selection of a target VOI in the case of follow-up studies on an approximately 65-year-old patient.

\section{Conclusions}

We proposed an automatic VBM software program of structural MRI for discrimination between patients with probable $\mathrm{AD}$ from the very-mild- to advanced stages and age-matched healthy controls. Application of the SPM8 plus DARTEL analysis to this software program provided a high accuracy of 91.6\% for discrimination of patients with very mild AD from healthy controls by using a target VOI located in medial temporal structures. Equal accuracies were obtained in early-onset and late-onset very mild AD subgroups. This software program may be useful for early diagnosis and longitudinal evaluation of $\mathrm{AD}$.

\section{References}

1. Frisoni GB, Fox NC, Jack CR Jr, et al. The clinical use of structural MRI in Alzheimer disease. Nat Rev Neurol 2010;6:67-77

2. Gómez-Isla T, Price JL, McKeel DW Jr, et al. Profound loss of layer II entorhinal cortex neurons occurs in very mild Alzheimer's disease. J Neurosci 1996;16:4491-500

3. Ashburner J, Friston KJ. Voxel-based morphometry: the methods. Neuroimage 2000;11:805-21

4. Baron JC, Chételat G, Desgranges B, et al. In vivo mapping of gray matter loss with voxel-based morphometry in mild Alzheimer's disease. Neuroimage 2001;14:298-309

5. Whitwell JL, Josephs KA, Murray ME, et al. MRI correlates of neurofibrillary tangle pathology at autopsy: a voxel-based morphometry study. Neurology 2008;71:743-49

6. Hirata Y, Matsuda H, Nemoto K, et al. Voxel-based morphometry to discriminate early Alzheimer's disease from controls. Neurosci Lett 2005;382:269-74

7. McKhann G, Drachman D, Folstein M, et al. Clinical diagnosis of Alzheimer's disease: report of the NINCDS-ADRDA work group under the auspices of Department of Health and Human Services taskforce on Alzheimer's disease. Neurology 1984;34:939-44

8. Petersen RC, Doody R, Kurz A, et al. Current concepts in mild cognitive impairment. Arch Neurol 2001;58:1985-92

9. Hughes CP, Berg L, Danziger WL, et al. A new clinical scale for the staging of dementia. Br J Psychiatry 1982;140:566-72

10. Ashburner J. A fast diffeomorphic image registration algorithm. Neuroimage 2007;38:95-113

11. Matsuda H, Mizumura S, Nagao T, et al. Automated discrimination between very early Alzheimer disease and controls using an easy Z-score imaging system for multicenter brain perfusion single-photon emission tomography. AJNR Am J Neuroradiol 2007;28:731-36

12. Kawachi T, Ishii K, Sakamoto S, et al. Comparison of the diagnostic performance of FDG-PET and VBM-MRI in very mild Alzheimer's disease. Eur J Nucl Med Mol Imaging 2006;33:801-09

13. Pereira JM, Xiong L, Acosta-Cabronero J, et al. Registration accuracy for VBM studies varies according to region and degenerative disease grouping. Neuroimage 2010;49:2205-15

14. Takahashi R, Ishii K, Miyamoto N, et al. Measurement of gray and white matter atrophy in dementia with Lewy bodies using diffeomorphic anatomic registration through exponentiated lie algebra: a comparison with conventional voxel-based morphometry. AJNR Am J Neuroradiol 2010;31:1873-78

15. Karas GB, Burton EJ, Rombouts SA, et al. A comprehensive study of gray matter loss in patients with Alzheimer's disease using optimized voxel-based morphometry. Neuroimage 2003;18:895-907

16. Matsunari I, Samuraki M, Chen WP, et al. Comparison of 18F-FDG PET and optimized voxel-based morphometry for detection of Alzheimer's disease: aging effect on diagnostic performance. J Nucl Med 2007;48:1961-70

17. Frisoni GB, Pievani M, Testa $C$, et al. The topography of grey matter involvement in early and late onset Alzheimer's disease. Brain 2007;130:720-30

18. Ishii K, Kawachi T, Sasaki H, et al. Voxel-based morphometric comparison between early- and late-onset mild Alzheimer's disease and assessment of diagnostic performance of $\mathbf{z}$ score images. AJNR Am J Neuroradiol 2005;26: $333-40$ 